\title{
Introduction to the Minitrack on Emerging Issues in Distributed Group Decision-Making: Opportunities and Challenges
}

\author{
Anil K. Aggarwal \\ University of Baltimore \\ USA \\ aaggarwal@ubalt.edu \\ Doug Vogel \\ Harbin Institute of Technology \\ PRC \\ isdoug@cityu.edu.hk
}

\author{
Yuko Murayama \\ Tsuda College \\ Japan \\ murayama@tsuda.ac.jp
}

This mini track addresses emerging issues, such as diversity, culture, adaptability, mobility, remote monitoring, smartphone vulnerability, herding and agility related to teams in distributed group decisionmaking, as well as the underlying theories of group dynamics, coordination, and communications among groups. The papers submitted specifically examined the emerging issues related to team configuration, herding, diversity, smart phone usage and gesture recognition system performance in a distributed environment.

The mini track attracted several papers related to various aspects of distributed decision making. Accepted papers study risk associated with smartphone, herding in virtual groups, social identity on decision making, diversity and conflict in teams and models for recurrent neural network as part of a strategy for the multi-agent patrolling task In the First paper authors discuss risk awareness of sites by giving users a sense of discomfort and helping them not to access dangerous sites. Authors studied discomfort feelings while using smartphones and extracted five discomfort factors from a questionnaire survey and factor analysis. Authors found three factors out of the five, namely, "Unintended operation or display," "Sudden changes," and "Understanding of the application," significant for risk awareness. In the second paper authors investigated the impact of social identity on decision-making in a distributed healthcare systems development team. Contrary to Social Identity Theory (SIT) authors found dissolution of distinct social groupings and rise of individualism within the project.
Based on their experiment they discover five inhibitors which can impede social identification in distributed teams: role ambiguity, absence of a collective vision, transfer of ownership, lack of shared history, and incompatible personalities. In third paper author discuss herding in virtual groups. Their study indicates that herding is dynamic and develops as levels of communication changes with incompetence and indifference of members. Authors present a three stage model to study herding progress over time. Authors report indifference and incompetence as two new factors that can lead to herding possibly leading to sub optimal results. In the fourth paper, author discuss the gesture recognition system based on a noncontact sensor based skeleton recognition system. Authors implemented several versions of gesture recognition systems by combining methods. The experimental results showed - Gesture Recognition System may be better separated into two components: One is a component that detects distinctive poses in Gestures, and the other is a component that detects the time-series appearance of them. - Distinctive poses should be selected flexibly.

Each of the above paper is timely, as they address emerging issues related to distributed group decisionmaking. The distributed group decision making area is still emerging and research is conflicting. As long as research produces mixed results, there will be continual need for validation and replication of experiments and development of new underlying theories. 